\title{
Bone Marrow Transplantation for Recessive Dystrophic Epidermolysis Bullosa
}

John E. Wagner, M.D., Akemi Ishida-Yamamoto, M.D., Ph.D., John A. McGrath, M.D., Maria Hordinsky, M.D., Douglas R. Keene, B.S., Megan J. Riddle, B.A., Mark J. Osborn, Ph.D., Troy Lund, M.D., Ph.D., Michelle Dolan, M.D., Bruce R. Blazar, M.D., and Jakub Tolar, M.D., Ph.D. Blood and Marrow Transplant Program, Department of Pediatrics (J.E.W., M.J.R., M.J.O., T.L., B.R.B., J.T.), Center for Translational Medicine (J.E.W., B.R.B., J.T.), and the Departments of Dermatology (M.H.) and Laboratory Medicine and Pathology (M.D.), University of Minnesota, Minneapolis; the Department of Dermatology, Asahikawa Medical College, Asahikawa, Japan (A.I.Y.); St. John's Institute of Dermatology, King's College London (Guy's Campus), London (J.A.M.); and Microimaging Center, Shriners Hospital for Children, Portland, OR (D.R.K.)

\section{Abstract}

Background-Recessive dystrophic epidermolysis bullosa is an incurable, often fatal mucocutaneous blistering disease caused by mutations in COL7A1, the gene encoding type VII collagen (C7). On the basis of preclinical data showing biochemical correction and prolonged survival in $\mathrm{col}^{-1}$ mice, we hypothesized that allogeneic marrow contains stem cells capable of ameliorating the manifestations of recessive dystrophic epidermolysis bullosa in humans.

Methods-Between October 2007 and August 2009, we treated seven children who had recessive dystrophic epidermolysis bullosa with immunomyeloablative chemotherapy and allogeneic stemcell transplantation. We assessed C7 expression by means of immunofluorescence staining and used transmission electron microscopy to visualize anchoring fibrils. We measured chimerism by means of competitive polymerase-chain-reaction assay, and documented blister formation and wound healing with the use of digital photography.

Results-One patient died of cardiomyopathy before transplantation. Of the remaining six patients, one had severe regimen-related cutaneous toxicity, with all having improved wound healing and a reduction in blister formation between 30 and 130 days after transplantation. We observed increased C7 deposition at the dermal-epidermal junction in five of the six recipients, albeit without normalization of anchoring fibrils. Five recipients were alive 130 to 799 days after transplantation; one died at 183 days as a consequence of graft rejection and infection. The six recipients had substantial proportions of donor cells in the skin, and none had detectable anti-C7 antibodies.

Conclusions-Increased C7 deposition and a sustained presence of donor cells were found in the skin of children with recessive dystrophic epidermolysis bullosa after allogeneic bone marrow transplantation. Further studies are needed to assess the long-term risks and benefits of such therapy in patients with this disorder. (Funded by the National Institutes of Health; ClinicalTrials.gov number, NCT00478244.)

Loss of Skin Integrity That Leads to trauma-induced blisters and erosions is a defining feature of epidermolysis bullosa, a heterogeneous group of more than 20 inherited blistering diseases

\footnotetext{
Address reprint requests to Dr. Wagner at the Division of Pediatric Hematology/ Oncology and Blood and Marrow Transplantation, University of Minnesota, Mayo Mail Code 366, 420 Delaware St. SE, Minneapolis, MN 55455, or at wagne002@ umn.edu. Drs. Blazar and Tolar contributed equally to this article.

Disclosure forms provided by the authors are available with the full text of this article at NEJM.org.
} 
with highly variable clinical severity. ${ }^{1}$ One of the most severe forms is recessive dystrophic epidermolysis bullosa, caused by loss-of-function mutations in the collagen type VII (C7) gene $(C O L 7 A 1) .^{2-6}$ These mutations result in severely diminished expression of $\mathrm{C} 7$, a collagen localized at the dermal-epidermal junction. $\mathrm{C} 7$ is the major component of the anchoring fibrils that tether the epidermal basement membrane to the dermal matrix. In the absence of normal C7 expression, these fibrils do not form properly, and epidermal-dermal adherence is lost beneath the lamina densa of the basement membrane.

From birth on, children with recessive dystrophic epidermolysis bullosa have painful erosions and blisters on mucosal membranes and skin, often resulting in esophageal strictures, mutilating scars, local and systemic infections, joint contractures, fusion of fingers and toes, and aggressive squamous-cell carcinomas. ${ }^{7-9}$ For patients with the Hallopeau-Siemens variant of recessive dystrophic epidermolysis bullosa, severe mucocutaneous disease is apparent at birth and the median survival is approximately 30 years, whereas persons with other types of recessive dystrophic epidermolysis bullosa have a median survival of 55 to 65 years. ${ }^{10}$

To date, the care of patients with recessive dystrophic epidermolysis bullosa has been palliative and restricted to the treatment of individual wounds. ${ }^{11,12}$ To assess the potential effectiveness of bone marrow stem-cell transplantation for the systemic correction of recessive dystrophic epidermolysis bullosa, we evaluated this approach in a mouse mode ${ }^{13}$ and observed that a stem-cell-enriched fraction of bone marrow prevented postnatal death in a proportion of mice with recessive dystrophic epidermolysis bullosa. Fifteen percent of the treated mice survived for longer than 80 days after transplantation; these mice had expression of wild-type $\mathrm{C} 7 \mathrm{in}$ skin and mucosa, formation of new anchoring fibrils, and resistance to blistering. ${ }^{14} \mathrm{We}$ then conducted a phase 1-phase 2 clinical trial of bone marrow transplantation for the treatment of severe recessive dystrophic epidermolysis bullosa.

\section{Methods}

\section{Patients and Treatment}

Between October 2007 and August 2009, we enrolled seven children with recessive dystrophic epidermolysis bullosa in a trial of immunomyeloablative chemotherapy and transplantation of allogeneic stem cells. Follow-up data are reported through January 8, 2010.

To optimize engraftment, we used a modified conditioning regimen for immunomyeloablation that consisted of busulfan $(0.8 \mathrm{mg}$ per kilogram of body weight per dose for children who weighed $12 \mathrm{~kg}$ or more and $1.1 \mathrm{mg}$ per kilogram for those who weighed less than $12 \mathrm{~kg}$, delivered intravenously every 6 hours on days 9 to 6 before transplantation, with dose modifications based on pharmacokinetics, targeting $1000 \mu \mathrm{mol}$ per minute for the area under the curve), fludarabine ( $25 \mathrm{mg}$ per square meter of body-surface area per day, given intravenously on days 5 to 3 before transplantation), and cyclophosphamide (50 mg per kilogram per day, given intravenously on days 5 to 2 before transplantation). (The treatment schema is shown in Fig. 1 in the Supplementary Appendix, available with the full text of this article at NEJM.org.) Immunoprophylaxis against graft-versus-host disease consisted of cyclosporine (targeting trough levels of 200 to $400 \mu \mathrm{g}$ per liter on day 3 before transplantation to day 100 after transplantation, with the dose tapered by $10 \%$ each week thereafter) and mycophenolate mofetil ( $15 \mathrm{mg}$ per kilogram on day 3 before transplantation to day 45 after transplantation).

On the day of transplantation, five patients received unfiltered marrow stem cells from an HLAidentical sibling; one of the five (Patient 1) concomitantly received umbilical-cord blood from the same sibling donor. Patient 3 received umbilical-cord blood from an unrelated donor (HLA-5/6, with B antigen mismatched). Owing to severe chemotherapy-related toxicity, one 
patient (Patient 2) did not receive the scheduled marrow infusion. Except for intensive skin care and placement of a gastrostomy tube to minimize vomiting, supportive care was standard for the recipients of an allogeneic bone marrow transplant. ${ }^{15}$ The study protocol was approved by the University of Minnesota institutional review board. Parents of all the study participants provided written informed consent, and Patient 7 provided assent.

\section{Laboratory Assessments}

To quantify and determine the location of C7, skin-biopsy specimens were obtained at perilesional sites on the extremities, approximately $1 \mathrm{~mm}$ away from an erosion, and were stained with anti-C7 antibodies. Six areas were selected at random locations along the dermalepidermal junction to quantify the $\mathrm{C} 7$ fluorescent-signal intensity (see the Methods section in the Supplementary Appendix).

Skin samples were also fixed and processed for transmission electron microscopy, as described previously. ${ }^{14}$ Qualitative and quantitative assessments, made at specific time points after treatment, focused on the development of anchoring fibrils beneath the lamina densa. Previously defined criteria for ultrastructural evaluation were applied ${ }^{16-18}$; specifically, the insertion of a fibrillar structure into the dermal surface of the lamina densa and central crossbanding, a fan-shaped appearance, or both were considered morphologic hallmarks of anchoring fibrils. Electron-microscopical images were reviewed independently by three dermatopathologists without knowledge of clinical outcome data.

We carried out fluorescence in situ hybridization (FISH) with the use of established methods (see the Supplementary Appendix) and determined the extent of blood and skin chimerism by means of competitive polymerase-chain-reaction analysis of polymorphic variable-number tandem-repeat regions. ${ }^{15} \mathrm{We}$ used a quantitative enzyme-linked immunosorbent assay to test for anti-C7 antibodies in blood samples. ${ }^{19}$ Images were scanned with the Epson Perfection V700 Photo Scanner, and densitometry was performed with the use of ImageJ software.

\section{Results}

\section{Patient and Graft Characteristics}

Six children with recessive dystrophic epidermolysis bullosa between the ages of 15 months and 14.5 years completed treatment and could be evaluated. Characteristics of the patients and grafts and the outcomes of transplantation are summarized in Table 1. Pretransplantation evaluations showed extensive cutaneous disease in each of the six patients and severe mucosal disease in four of the six, who required esophageal dilation and gastrostomy-tube placement for nutritional support. Five patients had severe mitten deformities, and four used wheelchairs. In addition, two had clinically significant renal impairment (glomerular filtration rate, 26.01 and $79.49 \mathrm{ml}$ per minute per $1.73 \mathrm{~m}^{2}$ of body-surface area in Patient 2 and Patient 4, respectively) and four had severe iron-deficiency anemia.

\section{Transplantation-Related Events}

Despite a $50 \%$ reduction in the dose of fludarabine because of a reduced glomerular filtration rate, Patient 2 died before the bone marrow infusion could be carried out as a result of hemorrhagic cardiomyopathy that was probably due to cyclophosphamide cardiotoxicity. Among the six patients who underwent transplantation, neutrophil recovery $\left(\geq 5 \times 10^{8}\right.$ cells per liter) occurred on days 11, 24, 16, 12, 14, and 18, in Patients 1, 3, 4, 5, 6, and 7, respectively, and platelet recovery ( $\geq 5 \times 10^{10}$ platelets per liter) occurred on days $43,33,46,37$, and 41 in Patients 1, 4, 5, 6, and 7, respectively, but was not achieved in Patient 3. Regimen-related toxicity included grade 3 mucositis (in six patients); transient, clinically significant hyperbilirubinemia (in four patients), with a peak total bilirubin level of 6.9 to $19 \mathrm{mg}$ per 
deciliter (118 to $325 \mu \mathrm{mol}$ per liter); renal insufficiency requiring 3 days of hemodialysis for fluid management (in two patients), with peak creatinine levels of $1.66 \mathrm{mg}$ per deciliter (146.7 $\mu$ mol per liter) in Patient 4 and $1.58 \mathrm{mg}$ per deciliter (139.7 $\mu$ mol per liter) in Patient 5; and opportunistic infections (candidemia in Patients 4 and 7 and viral reactivations in Patient 3 [adenovirus, Epstein-Barr virus, BK virus, and human herpesvirus type 6] and Patient 4 [BK virus]). No patient had acute or chronic graft-versus-host disease. Five patients were alive 799, 387, 268, 196, and 130 days after bone marrow transplantation, with Patients 1, 4, 5, and 6 having been taken off all immunosuppressive therapy and Patient 7 tolerating the tapering of the cyclosporine dose. Patient 3 , who required a second transplant because of graft rejection, had hematopoietic recovery (on day 22 after the second infusion of umbilical-cord blood) with complete marrow chimerism; however, subsequent infections resulted in his death on day 183.

\section{Assessment of Mucocutaneous Blistering and Wound Healing}

Mucocutaneous blistering was not adversely affected early after transplantation except in Patient 7 , in whom grade 4 cutaneous toxicity developed on day 17, presumably related to the chemotherapy regimen. All the patients had increased wound healing and decreased mucocutaneous blistering within the first 100 days after transplantation (Fig. 1). Patients 4, 5, and 7 had marked clinical improvement as early as day 30 . The percentage of body-surface area affected was reduced in Patients 1, 3, and 6 according to parents' reports and clinical observation, with more objective evidence provided by documented reductions in bandage use (assessed on the basis of the weight of precut dressings) in Patient 5 at 6 months and in Patient 4 at 1 year, as compared with bandage use before transplantation (Table 1). The parents of each patient reported substantial improvements in the rate of wound healing and marked reductions in oral mucosal lesions. We tested the skin of the two most recently enrolled patients (Patients 6 and 7) for resistance to blister formation on exposure to negative pressure. In both patients, the time to blister formation gradually increased after transplantation and exceeded that observed before transplantation (Fig. 2 in the Supplementary Appendix).

\section{Biochemical and Ultrastructural Assessments of Skin}

Skin-biopsy specimens were evaluated for the presence of C7 (Fig. 1). In Patient 1, skin immunofluorescence microscopy at baseline revealed faint linear and interrupted $\mathrm{C} 7$ immunolabeling at the dermal-epidermal junction; in contrast, by day 200 there was bright linear C7 immunoreactivity at the dermal-epidermal junction, which was even more intense by day 365 after transplantation. Skin-biopsy specimens from Patients 3, 4, 5, and 7 also revealed increases in $\mathrm{C} 7$ immunoreactivity at the dermal-epidermal junction after transplantation. In addition, we found that cultured fibroblasts from skin-biopsy specimens obtained after transplantation produced C7, as detected on Western blotting (data not shown).

Skin-biopsy specimens from all the patients except Patient 1 were evaluated with the use of six anti-C7 antibodies, which could potentially recognize different epitopes on wild-type C7. Testing with at least one anti-C7 antibody revealed a progressive increase in $\mathrm{C} 7$ expression as measured by increased fluorescence intensity over time in five of the six patients (Fig. 2); in Patient 6, C7 could not be detected at any point in time. $\mathrm{C} 7$ was either absent or barely detectable with at least one anti-C7 antibody before treatment in Patients 3 and 4; however, expression of $\mathrm{C} 7$ became readily apparent after transplantation and increased substantially over time.

Electron micrographs obtained at baseline revealed a complete absence of mature anchoring fibrils in all the patients. After transplantation, electron micrographs in all but one patient revealed only scanty, wispy structures under the lamina densa, as shown in Patient 7 (Fig. 3). Although this fibrillar material could represent very rudimentary anchoring fibrils or fragmented elastic microfibrils, more precise interpretation or reproducible quantification was not possible. In Patient 4, we observed occasional fibrillar structures below the lamina densa 
that were consistent with rudimentary anchoring fibrils (Fig. 3), but these structures were thin and generally lacked central cross-banding. After transplantation, all patients had an increase in the number of such structures, but none had the morphologic hallmarks of normal anchoring fibrils. Other ultrastructural changes included thickening of the lamina densa in Patients 4 and 5 (Table 1 and Fig. 3).

\section{Anti-C7 Antibody Evaluation}

Because anti-C7 antibodies could interfere with the benefit of transplantation, we assayed levels of anti-C7 antibodies in serum. Before transplantation, we detected low antibody titers in Patients 1, 3, and 7; no anti-C7 antibodies have been detected since transplantation.

\section{Chimerism Assessments}

After transplantation, all patients (including Patient 3, who required a second umbilical-cord blood transplant) had complete replacement of the marrow and blood by donor lymphohematopoietic cells. Moreover, all patients had evidence of donor cells in the skin, with a median of $20 \%$ (range, 0 to 93) being of donor origin. The percentage of donor cells varied between time points and with the biopsy site (Table 1). Patient 5 had a sex-mismatched donor. With the use of FISH to identify X and Y chromosomes (male donor), anti-CD45 and antiCD31 fluorochromes were used to determine the identity of the donor cells in the skin-biopsy specimens. We observed extravascular localization of CD45+ (hematopoietic) donor cells and CD45-CD31- (nonhematopoietic, nonendothelial) donor cells in the papillary dermis and epidermis in this patient (Fig. 3 in the Supplementary Appendix).

\section{Discussion}

We found that the infusion of allogeneic bone marrow can partially correct the $\mathrm{C} 7$ deficiency and improve skin and mucosal integrity in patients with recessive dystrophic epidermolysis bullosa. The observation that partial C7 correction is associated with a clinical benefit is consistent with previous work showing that a $\mathrm{C} 7$ level as low as $10 \%$ of the wild-type level of expression is associated with stability of the skin. ${ }^{20}$ The rates of recovery and ultimate outcomes varied among the surviving patients. Patients 4 and 5 had rapid and substantial clinical improvement relative to baseline, whereas Patient 1 had slow improvement, with only a modest overall benefit, and Patient 7 had a recurrence of blistering after an early period (days 30 to 60 ) of almost no blistering.

Unexpectedly, we detected substantial proportions of donor cells in the skin and mucosa after treatment; these proportions varied over time and with the location of the biopsy site. Many of the donor cells were located some distance away from blood vessels, and many were hematopoietic in origin (CD45+ cells). Although more work needs to be done to determine the identity of the CD45-CD31- donor cells, we favor the possibility that these healthy donor cells residing in the skin secrete $\mathrm{C} 7$ and that the secreted $\mathrm{C} 7$ is subsequently incorporated into the lamina densa at the dermal-epidermal junction.

The effect of new $\mathrm{C} 7$ on the morphologic features of anchoring fibrils, however, is not clear. Because all the patients except Patient 6 had mutant C7 expression at baseline (with Patients 4, 5, and 7 having higher levels of expression than Patients 1 and 3), it is possible that small amounts of newly formed C7 assembled with wild-type C7 early after transplantation to form a trimer and thus improved mucocutaneous integrity. This hypothesis also predicts that the $\mathrm{C} 7$ gene dose (i.e., the zygosity status of the donor) could influence the rate of improvement and is consistent with the blunted overall clinical outcome in Patient 1, who had a heterozygous donor with only one normal C7 gene. An initial scaffold of rudimentary anchoring fibrils in the host might be necessary for optimal deposition of wild-type C7 from the allogeneic cells, 
but it is clear that a clinical response does not depend on the presence of increased numbers of anchoring fibrils. Our data are limited to assessments of skin-biopsy specimens obtained within the first year after transplantation. Although rare anchoring-fibril-like structures were seen in Patient 4, the development of anchoring fibrils that fulfill the established ultrastructural criteria may require a longer interval after bone marrow infusion. Together, our findings suggest that the infusion of bone marrow from a healthy donor can ameliorate recessive dystrophic epidermolysis bullosa in humans, as has previously been shown in the mouse model of the disorder. Substantial efforts are under way to understand the physiology of the apparent clinical response after bone marrow transplantation and to identify the stem-cell population responsible for this effect.

Despite the apparent beneficial effect of bone marrow stem cells in patients with recessive dystrophic epidermolysis bullosa, allogeneic bone marrow transplantation remains a high-risk procedure typically reserved for patients with imminently life-threatening disease, such as those with high-risk lymphohematopoietic malignant disease. ${ }^{21}$ Before this clinical trial, it was not known whether patients with preexisting mucocutaneous disease could tolerate the conventional conditioning regimens used as preparation for allogeneic bone marrow transplantation. Although grade 3 mucositis occurred in all the patients in our study, as is typical for patients without preexisting mucocutaneous disease, only one patient had severe cutaneous toxicity. Notably, no patient had uncontrolled cellulitis, despite pretransplantation bacterial or fungal skin colonization. Clearly, the risks of bone marrow transplantation need to be weighed against the risks and benefits of alternative innovative therapies now being explored (e.g., injections of $\mathrm{C} 7$ protein or ex vivo grafting of allogeneic or gene-corrected autologous cells). $17,22^{-} 35$ Although these measures may prove to ameliorate morbidity, recessive dystrophic epidermolysis bullosa is a systemic disease, and the benefits of these other interventions have thus far been brief or limited to small areas of skin. Therefore, new approaches, such as the one we describe here, are being explored. Already, we and others are considering modifications to enhance safety, such as the coinfusion of mesenchymal stromal cells or the use of reduceddose conditioning before bone marrow transplantation, recognizing that the latter approach may increase the risk of opportunistic infection as a result of delayed immune reconstitution brought about by the additional immunosuppression.

In summary, our data suggest that the infusion of allogeneic bone marrow stem cells can lead to increased $\mathrm{C} 7$ deposition and reduced blistering in patients with severe recessive dystrophic epidermolysis bullosa. However, the unique skin and mucosal membrane defects of this disease pose a particular challenge to any bone marrow transplantation program. Clearly, much remains to be learned regarding the mechanism of the apparent functional correction as well as the longterm risks and benefits of this therapeutic approach, including the risk of squamous-cell carcinoma, which may occur after chemotherapy or as a result of incomplete correction of the underlying disease. Despite the potential benefits of marrow transplantation, it is a high-risk therapeutic approach that could shorten the expected survival of patients with recessive dystrophic epidermolysis bullosa, particularly those with less severe clinical manifestations. Nevertheless, the results to date support the study of stem-cell-based approaches for patients who have the severest forms of epidermolysis bullosa.

\section{Supplementary Material}

Refer to Web version on PubMed Central for supplementary material.

\section{Acknowledgments}

Supported in part by grants from the National Institutes of Health (5R01-HL049997, to Dr. Blazar), from the Ministry of Health, Labor, and Welfare of Japan and the Ministry of Education, Culture, Sports, Science, and Technology of 
Japan (to Dr. Ishida-Yamamoto), and from the University of Minnesota Academic Health Center, the Epidermolysis Bullosa (Liao Family) Research Fund, the Sarah Rose Mooreland EB Fund, and the Children's Cancer Research Fund.

We thank Dr. Angela M. Christiano for her helpful review of the original treatment protocol; Ron McElmurry, Brandon Peacock, Lily Xia, Amanda Kobs, Cindy Eide, Trevor Keyler, Christopher Lees, Carolyn Mann-Sandbakken, Meghan Munger, Michael Gitler, Dr. Ana Junqueira, Teresa Kivisto, and Pat Fidler for their technical and clinical help; Drs. Mei Chen and David Woodley for the anti-C7 antibody and for testing of anti-C7 antibodies in patients' blood before and after transplantation; and Noriko Takashita and Stomi Igawa for preparing the electron-microscopical images for the study.

\section{References}

1. Fine JD, Eady RA, Bauer EA, et al. The classification of inherited epidermolysis bullosa (EB): report of the Third International Consensus Meeting on Diagnosis and Classification of EB. J Am Acad Dermatol 2008;58:931-50. [PubMed: 18374450]

2. Dang N, Murrell DF. Mutation analysis and characterization of COL7A1 mutations in dystrophic epidermolysis bullosa. Exp Dermatol 2008;17:553-68. [PubMed: 18558993]

3. Wessagowit V, Kim SC, Woong Oh S, McGrath JA. Genotype-phenotype correlation in recessive dystrophic epidermolysis bullosa: when missense doesn't make sense. J Invest Dermatol 2005;124:863-6. [PubMed: 15816848]

4. Shimizu H, McGrath JA, Christiano AM, Nishikawa T, Uitto J. Molecular basis of recessive dystrophic epidermolysis bullosa: genotype/phenotype correlation in a case of moderate clinical severity. J Invest Dermatol 1996;106:119-24. [PubMed: 8592061]

5. Pulkkinen L, Uitto J. Mutation analysis and molecular genetics of epidermolysis bullosa. Matrix Biol 1999;18:29-42. [PubMed: 10367729]

6. Woodley DT, Hou Y, Martin S, Li W, Chen M. Characterization of molecular mechanisms underlying mutations in dystrophic epidermolysis bullosa using site-directed mutagenesis. J Biol Chem 2008;283:17838-45. [PubMed: 18450758]

7. Pillay E. Epidermolysis bullosa. 1. Causes, presentation and complications. Br J Nurs 2008;17:2926. Erratum, Br J Nurs 2008;17:413. [PubMed: 18414291]

8. Abercrombie EM, Mather CA, Hon J, Graham-King P, Pillay E. Recessive dystrophic epidermolysis bullosa. 2. Care of the adult patient. Br J Nurs 2008;17:S6, S8, S10. passim. [PubMed: 18521993]

9. Fine JD, Johnson LB, Weiner M, Li KP, Suchindran C. Epidermolysis bullosa and the risk of lifethreatening cancers: the National EB Registry experience, 1986-2006. J Am Acad Dermatol 2009;60:203-11. [PubMed: 19026465]

10. Fine, JD. Premature death in epidermolysis bullosa. In: Fine, JD.; Hinter, H., editors. Life with epidermolysis bullosa (EB): etiology, diagnosis, multidisciplinary care and therapy. New York: Springer-Verlag; 2009. p. 197-203.

11. Mellerio JE, Weiner M, Denyer JE, et al. Medical management of epidermolysis bullosa: Proceedings of the IInd International Symposium on Epidermolysis Bullosa, Santiago, Chile, 2005. Int J Dermatol 2007;46:795-800. [PubMed: 17651159]

12. Ly L, Su JC. Dressings used in epidermolysis bullosa blister wounds: a review. J Wound Care 2008;17:482, 484-6, 488. passim. [PubMed: 18978687]

13. Heinonen S, Männikkö M, Klement JF, Whitaker-Menezes D, Murphy GF, Uitto J. Targeted inactivation of the type VII collagen gene (Col7a1) in mice results in severe blistering phenotype: a model for recessive dystrophic epidermolysis bullosa. J Cell Sci 1999;112:3641-8. [PubMed: 10523500]

14. Tolar J, Ishida-Yamamoto A, Riddle M, et al. Amelioration of epidermolysis bullosa by transfer of wild-type bone marrow cells. Blood 2009;113:1167-74. [PubMed: 18955559]

15. Brunstein CG, Barker JN, Weisdorf DJ, et al. Umbilical cord blood transplantation after nonmyeloablative conditioning: impact on transplantation outcomes in 110 adults with hematologic disease. Blood 2007;110:3064-70. [PubMed: 17569820]

16. Tidman MJ, Eady RA. Evaluation of anchoring fibrils and other components of the dermal-epidermal junction in dystrophic epidermolysis bullosa by a quantitative ultrastructural technique. J Invest Dermatol 1985;84:374-7. [PubMed: 4039741] 
17. Wong T, Gammon L, Liu L, et al. Potential of fibroblast cell therapy for recessive dystrophic epidermolysis bullosa. J Invest Dermatol 2008;128:2179-89. [PubMed: 18385758]

18. Tidman MJ, Eady RA. Ultrastructural morphometry of normal human dermal-epidermal junction: the influence of age, sex, and body region on laminar and non-laminar components. J Invest Dermatol 1984;83:448-53. [PubMed: 6209345]

19. Chen M, Chan LS, Cai X, O'Toole EA, Sample JC, Woodley DT. Development of an ELISA for rapid detection of anti-type VII collagen autoantibodies in epidermolysis bullosa acquisita. J Invest Dermatol 1997;108:68-72. [PubMed: 8980290]

20. Fritsch A, Loeckermann S, Kern JS, et al. A hypomorphic mouse model of dystrophic epidermolysis bullosa reveals mechanisms of disease and response to fibroblast therapy. J Clin Invest 2008;118:1669-79. [PubMed: 18382769]

21. Armitage JO. Bone marrow transplantation. N Engl J Med 1994;330:827-38. [PubMed: 8114836]

22. Woodley DT, Keene DR, Atha T, et al. Injection of recombinant human type VII collagen restores collagen function in dystrophic epidermolysis bullosa. Nat Med 2004;10:693-5. [PubMed: 15195089]

23. Woodley DT, Remington J, Huang Y, et al. Intravenously injected human fibroblasts home to skin wounds, deliver type VII collagen, and promote wound healing. Mol Ther 2007;15:628-35. [PubMed: 17245357]

24. Remington J, Wang X, Hou Y, et al. Injection of recombinant human type VII collagen corrects the disease phenotype in a murine model of dystrophic epidermolysis bullosa. Mol Ther 2009;17:26-33. [PubMed: 19018253]

25. Mecklenbeck S, Compton SH, Mejía JE, et al. A microinjected COL7A1-PAC vector restores synthesis of intact procollagen VII in a dystrophic epidermolysis bullosa keratinocyte cell line. Hum Gene Ther 2002;13:1655-62. [PubMed: 12228020]

26. Chen M, Kasahara N, Keene DR, et al. Restoration of type VII collagen expression and function in dystrophic epidermolysis bullosa. Nat Genet 2002;32:670-5. [PubMed: 12426566]

27. Woodley DT, Keene DR, Atha T, et al. Intradermal injection of lentiviral vectors corrects regenerated human dystrophic epidermolysis bullosa skin tissue in vivo. Mol Ther 2004;10:318-26. [PubMed: 15294178]

28. Woodley DT, Krueger GG, Jorgensen CM, et al. Normal and gene-corrected dystrophic epidermolysis bullosa fibroblasts alone can produce type VII collagen at the basement membrane zone. J Invest Dermatol 2003;121:1021-8. [PubMed: 14708601]

29. Ortiz-Urda S, Thyagarajan B, Keene DR, et al. Stable nonviral genetic correction of inherited human skin disease. Nat Med 2002;8:1166-70. Erratum, Nat Med 2003;9:237. [PubMed: 12244305]

30. Ortiz-Urda S, Lin Q, Green CL, Keene DR, Marinkovich MP, Khavari PA. Injection of genetically engineered fibroblasts corrects regenerated human epidermolysis bullosa skin tissue. J Clin Invest 2003;111:251-5. [PubMed: 12531881]

31. Gache Y, Baldeschi C, Del Rio M, et al. Construction of skin equivalents for gene therapy of recessive dystrophic epidermolysis bullosa. Hum Gene Ther 2004;15:921-33. [PubMed: 15585108]

32. Ferrari S, Pellegrini G, Matsui T, Mavilio F, De Luca M. Gene therapy in combination with tissue engineering to treat epidermolysis bullosa. Expert Opin Biol Ther 2006;6:367-78. [PubMed: 16548763]

33. Ferrari S, Pellegrini G, Mavilio F, De Luca M. Gene therapy approaches for epidermolysis bullosa. Clin Dermatol 2005;23:430-6. [PubMed: 16023940]

34. Fivenson DP, Scherschun L, Choucair M, Kukuruga D, Young J, Shwayder T. Graftskin therapy in epidermolysis bullosa. J Am Acad Dermatol 2003;48:886-92. [PubMed: 12789180]

35. De Luca M, Pellegrini G, Mavilio F. Gene therapy of inherited skin adhesion disorders: a critical overview. Br J Dermatol 2009;161:19-24. [PubMed: 19466960] 


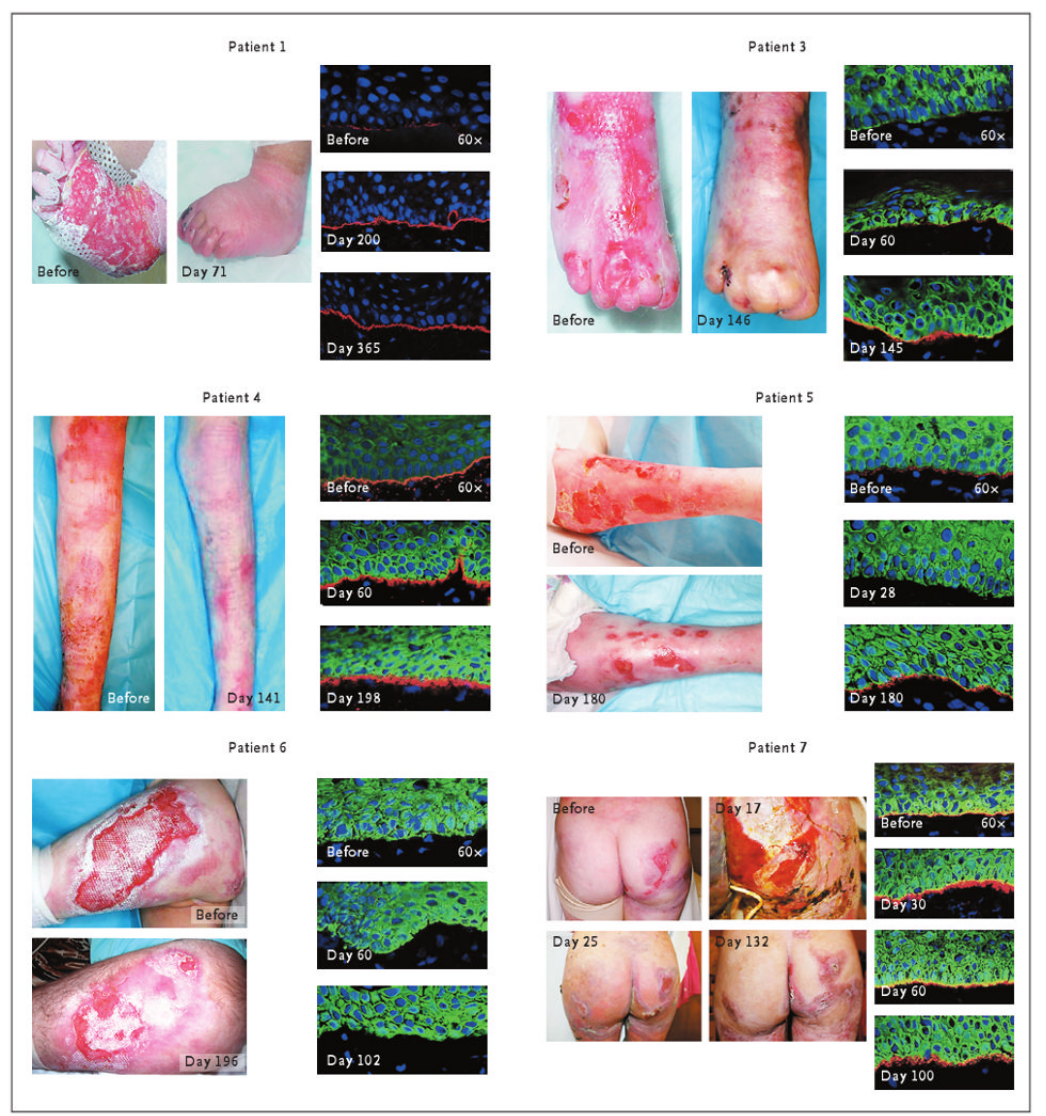

Figure 1. Clinical Photographs and Skin-Biopsy Specimens before and after Transplantation in Six Patients with Recessive Dystrophic Epidermolysis Bullosa

Photographs and skin-biopsy specimens were taken immediately before treatment and at scheduled time points after transplantation. The clinical photographs show specific areas that were consistently blistered before treatment. More rapid wound healing and reepithelialization were noted after transplantation in all six patients, with variable reductions in skin blistering. In Patient 7, marked cutaneous toxicity developed (presumably as a result of the chemotherapy) and was most pronounced on day 17 after transplantation but showed marked improvement by day 25. Immunofluorescence staining for $\mathrm{C} 7$ was carried out with skin-biopsy specimens obtained from all six patients, with the use of anti-C7 antibodies provided by Drs. D.T. Woodley and M. Chen (Keck School of Medicine, University of Southern California) for Patient 1 and purchased from BD Biosciences for the other five patients. For Patient 1, immunofluorescence staining before transplantation revealed faint, stippled labeling of C7; continuous, bright, linear C7 labeling was observed at all time points after bone marrow infusion. For Patient 3, faint, stippled C7 labeling was also observed at the dermal-epidermal junction before transplantation and through day 60 after transplantation, with continuous, bright, linear C7 labeling noted on day 145. For Patient 4, a bright, continuous band was observed at the dermal-epidermal junction before transplantation, with the use of anti-C7 antibody from BD Biosciences, whereas a thicker, brighter band was noted on day 198 after transplantation; this observation was confirmed with the use of LH7.2 anti-C7 antibody from Sigma-Aldrich, with no labeling before transplantation but bright labeling on day 198. For Patient 5, faint, stippled C7 labeling was observed at the dermal-epidermal junction with loss of demonstrable C7 labeling early after transplantation (day 28); however, continuous, bright linear, C7 labeling was observed on day 180. For Patient 6, no immunofluorescence staining was detectable before transplantation or at any time after transplantation, through day 102. For Patient 7, immunofluorescence staining 
before transplantation revealed stippled C7 labeling and continuous, bright, linear C7 labeling at the dermal-epidermal junction as early as day 30 after transplantation. For Patients 3 through 7 , keratinocytes (green) were visualized with the use of anti-cytokeratin 5 antibody. 


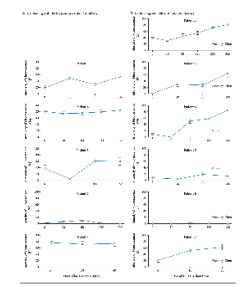

Figure 2. Mean $( \pm$ SE) Quantification of C7 Fluorescence Intensity at Specific Time Points after Transplantation

Panel A shows the relative fluorescence intensity with the use of an anti-C7 antibody from BD Biosciences. Panel B shows the results with the use of additional anti-C7 antibodies, which in some cases failed to bind mutant $\mathrm{C} 7$ before transplantation but did bind $\mathrm{C} 7$ after transplantation. An anti-C7 antibody provided by Drs. D.T. Woodley and M. Chen (Keck School of Medicine, University of Southern California) was used for Patients 1, 3, 6, and 7), and an anti-C7 antibody (LH7.2) made by Sigma-Aldrich was used for Patients 4 and 5. (Each antibody was applied to tissue from Patients 3 through 7; the data presented are for samples showing the most pronounced changes in C7 expression.) Species-specific and isotype-specific control antibodies, which were used in assays performed on the same day as those carried out with anti-C7 antibodies, yielded negative results (data not shown). Note that $100 \%$ is the maximal measurable fluorescence intensity on the gray scale. 


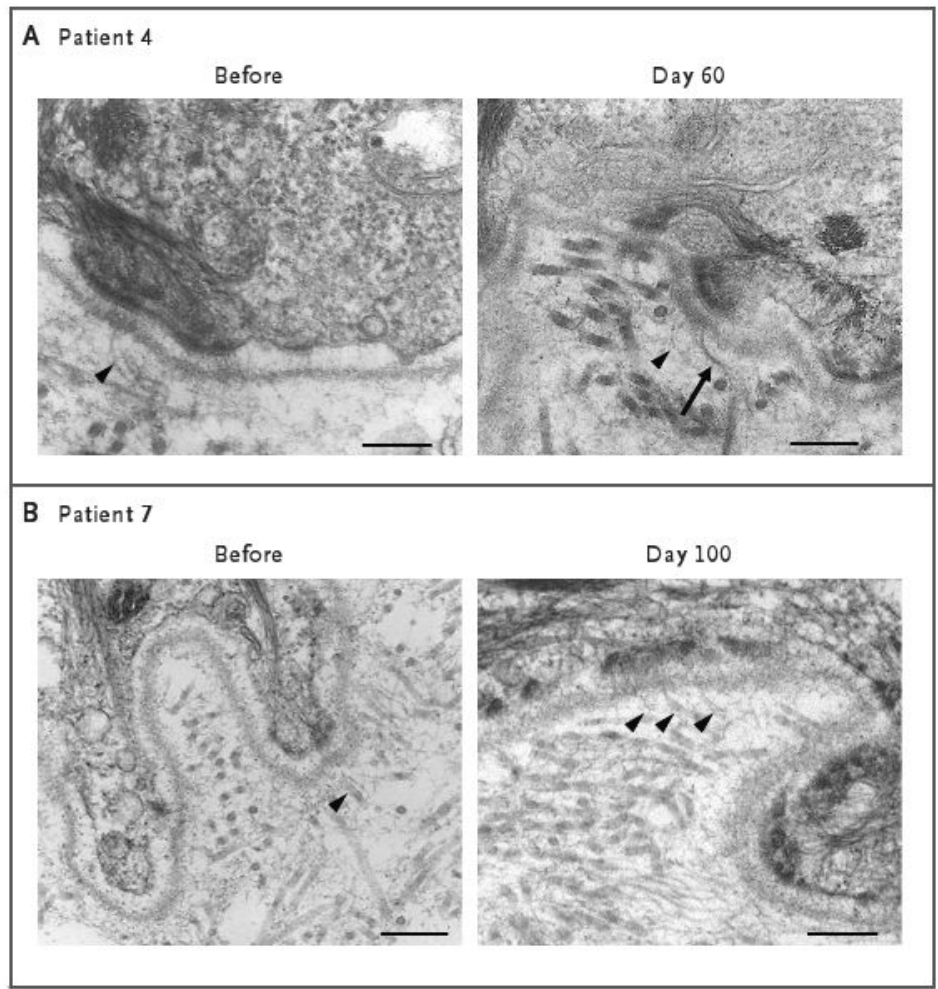

Figure 3. Transmission Electron Micrographs Showing Fibril Formation in Skin Specimens from Patients 4 and 7

Photomicrographs show thin fibrillar structures beneath the lamina densa before transplantation and on day 60 after transplantation in Patient 4 (Panel A, arrowheads) and before transplantation and on day 100 in Patient 7 (Panel B, arrowheads), with an increase in the number of fibrils after transplantation. Although some of these fibrils appear somewhat thicker in Patient 4 than in Patient 7 (Panel A, arrow), none bear the ultrastructural hallmarks of normal anchoring fibrils. The bars represent $250 \mathrm{~nm}$. 


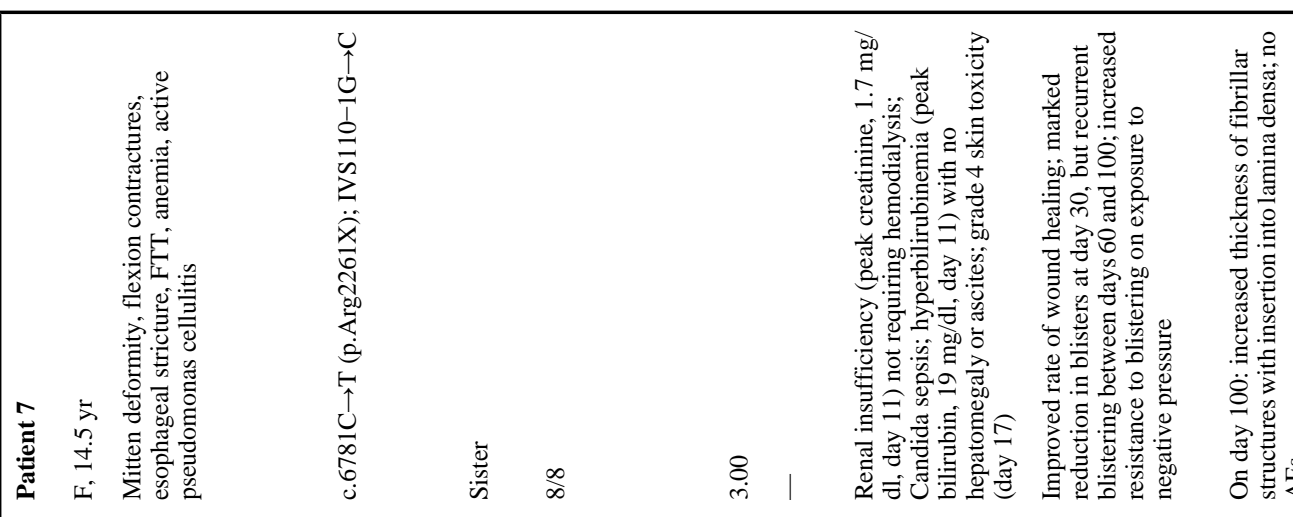

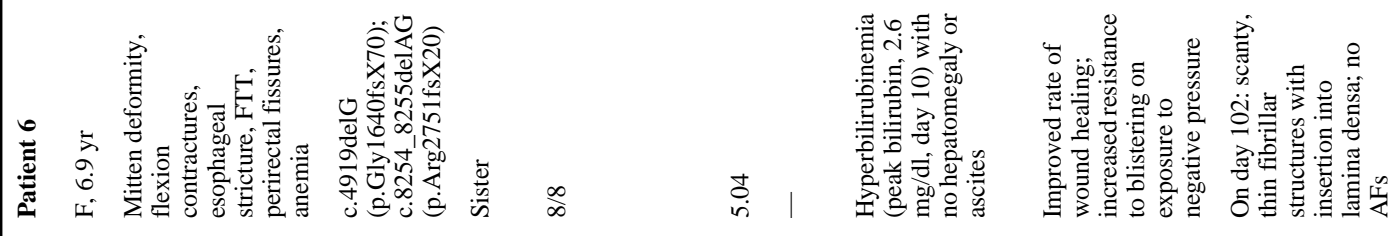
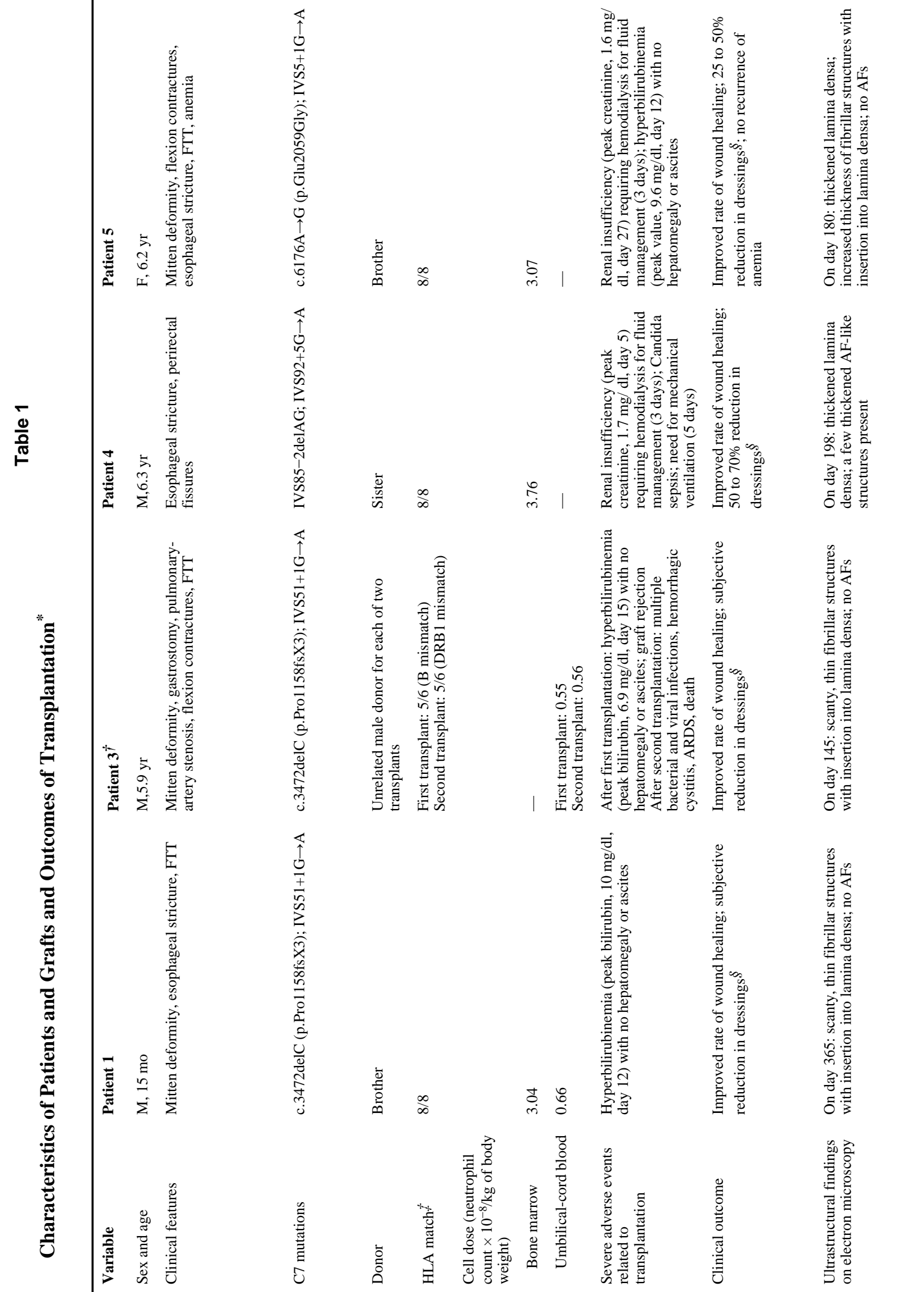

N Engl J Med. Author manuscript; available in PMC 2011 February 1. 


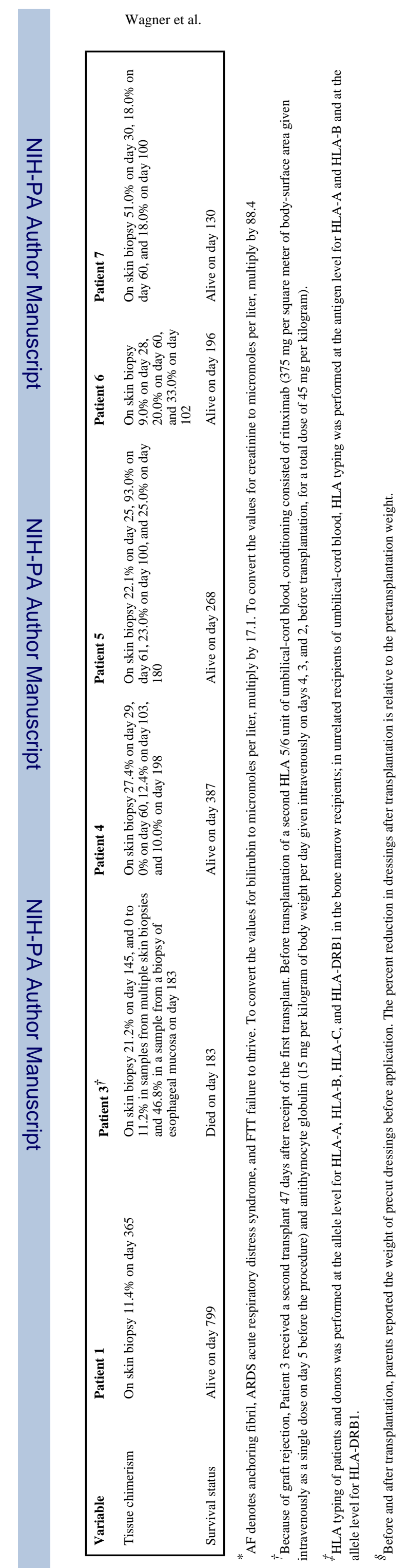

Page 14 